\title{
Research on Vehicle Routing problem Based on Genetic Algorithm
}

\author{
Ke Bai \\ Henan Economy and Trade Vocational College
}

\author{
Keywords: Vehicle Routing Problem ;Improved Genetic Algorithm
}

\begin{abstract}
This paper analyzed Vehicle Routing Problem and established mathematics former. After that it introduced the method and process of Simple Genetic Algorithm which was used to solve Vehicle Routing Problem. Then, it indicated the shortage of Simple Genetic Algorithm and put forward Improved Genetic Algorithm based on the coding project of second layer chromosome and adapted cross probability. Lastly, it validated the feasibility and validity of Improved Genetic Algorithm by test.
\end{abstract}

\section{Introduction}

Logistics has showed powerful function since it generated. As the groundwork of economic activity, logistics is known as the third profit source of enterprise. It can meet the requirements of modern business, and promote progress of the society and economy. So, it is important to promote the development and progress of logistics. Logistics has resulted in more and more attention.

In the costs of logistics, transportation cost accounts for a very high percentage. Logistics distribution derived from the transportation in logistics system, it is an important link of logistics. The scheme of logistics distribution has great influence in logistics service, costs and benefit in delivery whether it is reasonable. Vehicle Routing Problem is the core of logistics distribution and one of the focus of research. Reasonable arrangement can facilitate the procedure of distribution, reduce the time of distribution, improve transport efficiency and reduce service costs effectively. So, it is necessary to do some research on Vehicle Routing Problem.

At present, the research on VRP mainly focuses on the improved intelligent optimization algorithms.William etc.[1] proposed two kinds of hybrid genetic algorithm to solve multiple-depot vehicle routing problem. Mirabi etc. [2] used random hybrid heuristic algorithm to solve the VRP. Nilay etc. [3] adopted the genetic algorithm based on clustering technology to solve VRP. Zou Tong etc.[4] presented the VRP model, and used genetic algorithm to solve the problem. In order to optimize the goal, Liying Zheng etc.[5] did not consider the customer orders attributes and customer satisfaction in the optimization process. Along with the increase of the customer's number and the problem size, the solving efficiency of all kinds of existing heuristic algorithm must drop rapidly. In view of the actual large-scale VRP, greatly reducing the problem solving space becomes one of the effective ways to solve the problem.

\section{The basic principle of genetic algorithm}

Genetic algorithm is a kind of algorithm of random probability iterative search, which is based on Darwin's classical theory of "natural selection, survival of the fittest". The basic idea is the genetic variation of species evolution applied to calculating the optimal solution. Being left in the process of evolution is the most adaptable to environment, and the calculation for iteration can obtain far the most optimal solution.

Genetic algorithm is based on the population formed by potential solutions to resolve problems as a starting point, and each population contains a certain number of individuals, and these individuals are encoded by genes, making individuals into a chromosome of characteristics of individual entities. Genetic algorithm simplifies coding work and based on "survival of the fittest, survival of the fittest" theory, it continuously iterates and evolves better approximate solutions. Select individual is decided by individual fitness function of the problem domain and applies genetic operators to achieve crossover and mutation, and produce new stocks set until the iteration 
works out the best individual, that is, it finds the approximate optimal solution.

Genetic algorithm is mainly used to choose, crossover and mutation operations form. It is the first to random initialization of a certain amount of the parent individual, and gets its individual fitness function. In accordance with the optimizing principles, evolution produces new offspring, according to the fitness function choice and cross parent individuals generates new individuals. Offspring is to implement mutation. Then offspring fitness is again calculated, which is cycled until the best individual is produced, that is, the optimal solution of the problem.

\section{Improvement of genetic algorithm}

The two-stage fuzzy clustering forms the distribution customer base, the follows uses the proposed improved genetic algorithm to do vehicle routing optimization for the distribution customer base. This article improves the selection operator and crossover operator of traditional genetic algorithm, and the global optimization ability of this algorithm is improved. Improved selection operator will combine the best strategy and the roulette selection model, at the same time keeping the optimal solution ensures the fair opportunity that the rest solutions enter into the next generation, namely the bigger is the fitness value in the rest solutions, the bigger is the opportunity entering into the next generation.

The improved crossover operator randomly selects gene sections for cross through the front, and shifts the parent residual gene in sequence to form new progeny, to effectively avoid the local optimum.

(1) To generate the initial population. Chromosome encoding adopts the ordinal number coding

method based on customer, chromosome $\mathrm{G}$ is expressed by vector $\left(l_{1}, l_{2}, \ldots, l_{n}\right)$, gene $l_{i}$ is a natural number without repetition between $[1, n], n$ is the customer number, the random sequence of chromosome structure generates the initial population with size of $N_{1}$.

(2) Fitness function. The individuals with high fitness in genetic algorithm have greater probability to enter into the next generation, due to the objective function $f(x)$ is to solve the minimum path cost, the fitness function is expressed by the reciprocal of the objective function, that is, $f=1 / f(x)$.

(3) The operator selection. The selection strategy adopts the combination of retaining the best and roulette wheel selection, $N_{1}$ individuals in each generation sort according to the fitness value f, the individual ranked at No. 1 directly copies and goes into the next generation, lists side by side at the first place, the $\left(N_{1}-1\right)$ other individuals of the next generation will generate by using the roulette method, according to the fitness of $N_{1}$ individuals in the last generation. While keeping the best individual at the same time, the individuals with bigger fitness have greater chance to enter into the next generation.

(4) Crossover operator. Randomly generate two intersection points between two parents' generation (parent 1 and parent 2) respectively, the gene between two junctions is taken as gene section for cross, which are remarked as $(C-G E)_{1}$ and $(C-G E)_{2}$, progeny 1 takes $(C-G E)_{2}$ as the start gene, from left to right moves the non-overlap genes with ${ }^{(C-G E)_{2}}$ in parent 1 to the progeny1, similarly the progeny 2 can generate. Adopting the method of front cross gene sections, even if both of the parents are same, also can produce a new progeny to do iterative optimization, and overcome the shortcomings of local optimal and premature convergence.

(5) The mutation operator. Using translocation mutation operation, with a certain mutation probability randomly select the chromosomes with mutation, on the chromosomes randomly select two genes and swap the genes. 


\section{The result simulation}

Matlab 7.8 programming is used to realize random examples simulation, the program running environment is: $2.26 \mathrm{GHz}$ Intel CPU, $512 \mathrm{MB}$ memory. Within the Cartesian plane of 200x200, 62 customer points and three depots are randomly generated as shown in figure 1 . The attribute information of 62 customer order requirement is randomly generated, the quantity demand of each customer obeys the uniform distribution $U[1,10]$, the delivery deadline is randomly selected in $\{0.5,1,1.5,2,3,3.5,2.5,4\}$, the product warranty is generated from $\{30,45,60,120,180\}$, the customer demand for service quality and product exterior characteristic are randomly generated from \{very high, high, low, very low\}, suppose that the credibility of the data provided by the customer is 1 , when the fuzzy similarity calculation is executed, the delivery deadline, product warranty, customer demand for service quality and product exterior characteristic, the corresponding weights of these four decision variables are set to 0.3, 0.2, 0.3 and 0.2 , the similarity criterion $\lambda$ value is 0.84 , the division result of customer bases in the two-stage fuzzy clustering algorithm is shown in table 1

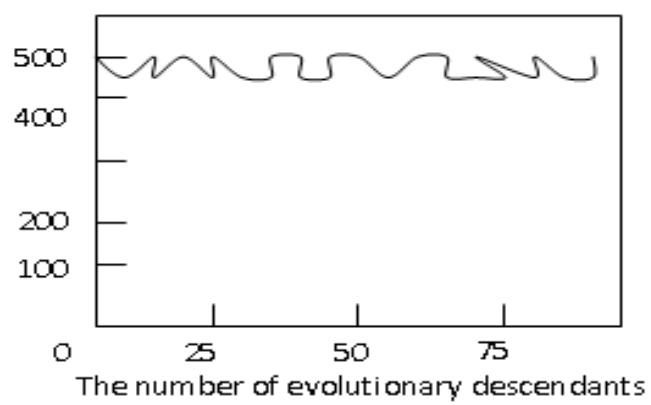

Figure 1. The distribution diagram between depots and customers

Table 1. The divisions result of customer bases in the two-stage fuzzy clustering algorithm

\begin{tabular}{|l|l|l|l|}
\hline $\begin{array}{l}\text { Upper } \\
\text { level } \\
\text { static } \\
\text { clustering }\end{array}$ & $\begin{array}{l}\text { Low level } \\
\text { fuzzy } \\
\text { clustering }\end{array}$ & $\begin{array}{l}\text { Distributed } \\
\text { customer base }\end{array}$ & $\begin{array}{l}\text { Vehicle } \\
\text { distance } \\
\text { optimizati } \\
\text { on }\end{array}$ \\
\hline F1 & $\begin{array}{l}\text { F11 } \\
\text { F12 }\end{array}$ & $\begin{array}{l}1,2,3,4,14,16,18 \\
7,11,15,17,19,20\end{array}$ & C,A,B \\
\hline F13 & F21 & $\begin{array}{l}5,6,8,9,10,12,13 \\
21,22,29,34,36,42,\end{array}$ & \\
& F22 & $\begin{array}{l}\text { 50,51,2,56,61 } \\
24,25,32,40,41,59\end{array}$ & A,B,C \\
& F31 & $\begin{array}{l}23,30,43,45,54,55, \\
57 \\
\text { F3 }\end{array}$ & B,A,C \\
& F32 & $\begin{array}{l}26,27,33,39,46,47 \\
28,31,35,37,38,44,\end{array}$ & \\
& F33 & & \\
& & $53,58,60,62$ & \\
\hline
\end{tabular}

According to the division results of customer base in table 2, the improved genetic algorithm is used for vehicle routing optimization. The model of distribution vehicle is single, the maximum loading capacity is 20t, and the improved genetic algorithm parameters are set to: the population size is 50 , the iteration time is 100 , the crossover probability is 0.9 , the mutation probability is 0.05 , 


\section{Conclusion}

With the rapid development of computer science the vehicle routing problem (VRP) is always the researching focus of most computer scholars. It is of great significance for the logistics system and the combination optimization problems that how to design the algorithm with simple operation and excellent capability according to the characteristics of the vehicle routing problem. Thus, the theory in vehicle routing is applied to two typical algorithms according to the shortage of the common genetic algorithm for VRP. Examples verify the effectiveness of the proposed algorithm, it not only can solve the genetic algorithm which is easy for the phenomenon of cyclic redundancy, also can solve the slowing down defects of loop iteration in the early period of the traditional algorithm to search , has a good application in practice.

\section{References:}

[1] Ho W., Ho. G T S, Ji P. A hybrid genetic algorithm for the multi-depot vehicle routing problem[J].Engineering Applications of Artificial Intelligence, 2008, 21(4): 548-557.

[2] Mirabi M, Ghomi S M T F, Jolai F. Efficient stochastic hybrid heuristics for the multi-depot vehicle routing problem. Robotics and Computer-Integrated Manufacturing, 2010, 26(6): 564-569.

[3] Yvcenur G N, Demirel N C. A new geometric shape-based genetic clustering algorithm for the multi-depot vehicle routing problem. Expert Systems with Applications, 2011,38(9): 11859-11865.

[4] Tong Zou, Ning Li, Debao Sun. The genetic algorithm of multiple-depot vehicle routing problem. Computer engineering and application, 2004, 40 (21): 81-83.

[5] Liying Zheng, Haipeng Jia. The multiple-depot heterogeneous-vehicle scheduling algorithm based on global search clustering. Journal of Lanzhou Jiao Tong university, 2009, 28 (6) : 19-22. 